\title{
Modelo de soporte técnico para la gestión de servicios tecnológicos en la administración pública nacional
}

\author{
Fecha de recepción: 04/12/2019 • Fecha de aceptación: 15/01/2020 • Fecha de publicación:10/02/2020
}

\author{
Paola Elizabeth Torres Regalado \\ Agencia de Regulación y Control Minero (ARCOM) \\ pao-Itr@hotmail.com \\ https://orcid.org/0000-0002-6049-946X
}

\section{RESUMEN}

Esta investigación propone un modelo de Soporte Técnico para la Gestión de Servicios Tecnológicos en la Superintendencia de la Información y Comunicación (SUPERCOM). A lo largo del estudio se ha podido identificar fortalezas, así como debilidades, entre las que se puede enfatizar la escasez de una planificación estratégica, índices de medición para evidenciar el trabajo tecnológico, información unificada en un solo gestor de aplicaciones, inadecuado tiempo de respuesta, entre otras inexactitudes.

Este análisis ha permitido establecer un modelo de gestión que consigue mejorar el tiempo de respuesta, eficacia y calidad de los servicios. Por tanto, se ha realizado una propuesta de un modelo de gestión integral que considera tanto los aspectos administrativos que generan falencias, así como un modelo operativo para el mejoramiento del servicio de TI, a través del cual será posible lograr mejores resultados, eficiencia y un desempeño óptimo de los servicios tecnológicos. El planteamiento de este modelo, basado en las mejores prácticas con una documentación consistente, comprensiva y no propietaria, para el manejo de servicios de TI, consolida diferentes aspectos estratégicos y operacionales de la organización, que permite al acceso del modelo libremente a diferencia de otras metodologías. 


\section{ABSTRACT}

This research proposes a model of Technical Support for the Management of Technological Services in the Superintendence of Information and Communication (SUPERCOM). Throughout the study it has been possible to identify strengths, as well as weaknesses, among which the shortage of strategic planning, measurement indices to highlight technological work, unified information in a single application manager, inadequate response time can be emphasized, among other inaccuracies.

This analysis has allowed us to establish a management model that improves the response time, efficiency and quality of services. Therefore, a proposal has been made for a comprehensive management model that considers both administrative aspects that generate failures, as well as an operational model for the improvement of the IT service, through which it will be possible to achieve better results, efficiency and optimal performance of technological services. The approach of this model, based on best practices with consistent, comprehensive and non-proprietary documentation, for the management of IT services, consolidates different strategic and operational aspects of the organization, which allows access to the model freely as opposed to others methodologies 


\section{INTRODUCCIÓN}

Las tecnologías de la información TI se han hecho cada vez más trascendentes en el desarrollo operacional y estratégico de las organizaciones, siendo una herramienta cada vez más indispensable en las actividades diarias, que al no gestionarlas adecuadamente pueden provocar: proyectos de TI truncados, pérdida de competitividad, gastos innecesarios de dinero, una mala gestión del talento humano, la no existencia de procesos y procedimientos, clientes insatisfechos, entre otros efectos.

Con estos antecedentes las empresas de todo el mundo han adoptado marcos de referencia conocidos como "Mejores prácticas" de la industria tecnológica para la gestión por procesos de los servicios de tecnología, los mismos que han evolucionado en los últimos 30 años desde su primera aparición, entre las más destacables se encuentran los estándares de principio como: COBIT (Control Objetives for information Systems and relational technology) un marco de referencia para la gobernabilidad de TI basándose en el control y auditoria de sistemas de información e ITIL (Information Technology Infrastructure Library) como un marco de referencia para la gestión de los servicios de tecnologías a través de procesos. También se han propuesto estándares de calidad de servicios como la norma ISO 9001 y la norma para la gestión de servicios de TI como ISO/IEC 20000. (ISO, 2017)

En base a lo mencionado, el uso normado de TIC se ha convertido dentro de una organización en un eje transversal para fortalecer los procesos de enseñanza y aprendizaje, facilitando el correcto funcionamiento de los procesos que se realizan en la organización.

La Superintendencia de la Información y Comunicación creada en el año 2013, según su directriz política propone: "ser un organismo técnico del Estado Ecuatoriano, que tiene capacidad constitucional y legal de velar por la vigilancia, auditoría, intervención y control de las actividades comunicacionales de producción y difusión de contenidos, a través de la radio, televisión, prensa y de las páginas web, registradas en Ecuador". (SUPERCOM), en el año 2017 - 2018.

La SUPERCOM, desde su inicio ha crecido de manera acelerada, aumentando su estructura física, su personal y la infraestructura tecnológica para poder atender el acelerado incremento de requerimientos de servicios tecnológicos. Resultado de esta gestión se encuentra una organización con una gran cantidad de servicios derivados del uso de las aplicaciones e infraestructura implementadas, no es menos cierto que este crecimiento ha sido desordenado, no se cuenta con procesos y procedimientos definidos ni formalizados, no existen estándares ni normas, tampoco existe referencias para hacer una gestión adecuada del área.

A mediano y largo plazo se deteriorará la calidad de los servicios creados si no se toma una acción correctiva la cual los servicios tecnológicos como los que se menciona a continuación:

- No identificar índices de medición para evidenciar el trabajo tecnológico.

- Manejo inadecuado de información. 
- No tener unificada la información en un solo gestor de aplicaciones.

- Tiempos de respuesta alta.

- Gastos elevados para desarrollo de sistemas.

Esta investigación, pretende determinar la situación actual de la SUPERCOM y proponer la estandarización de los procesos $\mathrm{TI}$, métricas para su control y mejoramiento de tiempos de respuestas, planes para mantener la integridad, disponibilidad y seguridad de la información, etc. EL objetivo principal de este proyecto es que la SUPERCOM se convierta en un ente replicador para los organismos de la Administración Pública Nacional con una mejoría en la gestión de los servicios de TI. Basado en un diseño e implementación de un módulo de soporte técnico para la gestión de los servicios tecnológicos considerando como perspectiva metodológica. (SUPERCOM, 2017)

\section{TEORÍA Y CONTEXTO}

El contexto teórico dentro de los estudios realizados a la problemática planteada para el desarrollo de la investigación. Se ha tomado en cuenta proyectos realizados que sirvieron de base como guía para el levantamiento de los objetivos, el marco metodológico sirvió como apoyo en el procesamiento y análisis de la información.

Sánchez (2016), plantea un estudio de la metodología ITIL, centro de educación continua CECIAEN, el cual pretende enseñar de una organización, cuáles son los beneficios de llevar desde una perspectiva funcional a una gestionada por procesos los cuales implican la descripción, el funcionamiento y aplicaciones, los cuales sirvió de apoyo para reforzar la base teórica de esta investigación, se diferencia que se desarrolla e implementa una herramienta basada en la metodología ITIL.

Fernández \& Llorens (2011), plantea descubrir cuál puede ser la importancia de gobierno de las TI para las universidades españolas, a partir de un análisis profundo de la literatura y en particular del estudio de experiencias llevadas a cabo en el ámbito universitario. Donde busca seleccionar o diseñar un modelo de gobierno de las TI que se convierta en referencia global para el sistema universitario español, las cuales sirvió de apoyo para complementar las bases teóricas y para la formulación de los modelos propuesto, se diferencia en el diseño e implementación.

Así como bases teóricas de definición de proceso, definición de gestión por procesos, gestión de $\mathrm{TI}$, gobierno de TI, estructura organizacional, cobit 4.1, modelo de madurez TI, ITIL V3, (Bergholz, 2011).

\section{MÉTODO}

El proyecto tiene una investigación de tipo documental y de campo que incluye ambas circunstancias. Se usó el método de investigación mixta ya que se utilizó un enfoque multidisciplinar, por lo tanto, se usaron los métodos cuantitativos y cualitativos conjuntamente, 
identificando los más idóneos en función de la naturaleza, los objetivos, la temática y el presupuesto del estudio, esto aplica a la observación de los sistemas de actividad humana llevados a cabo durante la resolución de un problema, donde el investigador esta activamente involucrado, con expectativas de beneficios tanto para el como para la organización.

En tal sentido, se presentan, las fases del método de investigación de los procesos de servicios tecnológicos de información basado en ITIL ya que COBIT contiene un conjunto amplio de procesos y lineamientos para una adecuada administración de TI, que se vuelve complejo de ser aplicada en empresas pequeñas debido a que no existen ni los recursos ni la identificación de los procesos.

Se exponen las etapas de cada una de las fases utilizadas en el presente de investigación, cuyo objetivo es crear un diagnóstico, revisión, levantamiento de información, adaptación, acción y diseño con una previa autorización de la máxima autoridad. Por lo que se genera variables para cumplir con los objetivos establecidos en esta investigación, como son:

- Salidas de información cuyo objetivo es describir los flujos de información de los procesos de Tecnología de Información de la SUPERCOM.

- Gestión de procesos comparar la situación actual de la gestión de procesos de tecnología de información en la SUPERCOM en relación con las disciplinas que propone ITIL.

- Modelo elaborar un modelo para la mejora de los procesos se servicios de tecnologías de información basado en las disciplinas ITIL, para la SUPERCOM.

\section{Análisis de la Situación Actual}

La dirección de tecnologías e información es la encargada de la entrega de los servicios relacionados a la infraestructura tecnológica y desarrollo de nuevas aplicaciones y sistemas informáticos para las diferentes áreas, por tanto, la encargada de realizar el levantamiento de información y análisis de los procesos presentes en la SUPERCOM, fruto de lo cual se identificó las oportunidades, debilidades y amenazas, que se describen a continuación:

\section{Oportunidades}

Apoyo por parte de las autoridades para el acceso a la información requerida, de forma que se incorpore nuevas tecnologías para obtener una mejor calidad en la gestión de los servicios de TI.

\section{Debilidades}

- Falta de planificación estratégica y de indicadores de gestión del servicio tecnológico.

- Falta de eficiencia en la aplicación óptima de los servicios, es decir, ausencia de procesos y un modelo de gestión que genera falta de efectividad en la prestación de los servicios de TI. 
- No existe una base de datos actualizada y centralizada.

\section{Amenazas}

- Percepción de ineficiencia en los resultados de la dirección de TI por las autoridades.

Uno de los aspectos importantes para realizar el diagnóstico de la SUPERCOM es determinar cuáles son los procedimientos que actualmente tiene la dirección de tecnologías de la SUPERCOM, puesto que serán analizados y evaluados a fin de conocer cuál es el grado de madurez de los servicios tecnológicos entregados.

Con el fin de profundizar en el análisis de la situación actual de la SUPERCOM, se ha utilizado como una de las herramientas de la investigación, la observación; misma que tendrá como objetivo conocer el nivel de satisfacción que tiene el personal de la SUPERCOM matriz, quienes podrían tener una opinión bastante acertada de la situación actual en relación al servicio, ya que, son usuarios de los mismos.

Al estudiar el nivel de servicio que se brinda a través del área de soporte técnico de la SUPERCOM, para la solución de problemas solicitados, en su mayoría se puede observar que el tiempo de solución es alto, sin evidenciar el trabajo tecnológico que con lleva para la solución, así como la falta de procesos y un sistema que pueda optimizar el tiempo de respuesta.

También se nota un tercer aspecto a mejorar, el monitoreo en la entrega de servicios, por lo tanto, es claro que se deben definir planes de acción que permitan mejorar el objeto mismo de la SUPERCOM, así como la posibilidad de dar solución a los problemas con eficiencia y eficacia, aspectos esenciales para ofrecer un servicio de calidad.

\section{Propuesta}

Luego del diagnóstico situación actual realizado en la SUPERCOM, fue posible identificar que existen falencias en la prestación de servicio de TI, por tanto, la necesidad de implementar un modelo de soporte técnico para la gestión de servicios tecnológicos sobre la base de modelos de gestión de la metodología ITIL, los cuales se han propuesto adaptar para el desarrollo e implementación que deberían existir en un departamento de tecnología de la información con el fin de alcanzar la excelencia operativa en la gestión de servicio de TI.

Se presenta las principales falencias encontradas y analizadas a través del diagnóstico previamente realizado y sus soluciones. 
Tabla 1. Falencias encontradas

\begin{tabular}{|c|c|}
\hline FALENCIAS & SOLUCIÓN ESPECÍFICA \\
\hline $\begin{array}{l}\text { Tiempos de espera para } \\
\text { soporte técnico }\end{array}$ & $\begin{array}{l}\text { Aplicación de módulo de gestión } \\
\text { de servicio tecnológica }\end{array}$ \\
\hline Indicadores de Gestión & $\begin{array}{l}\text { Aplicación de módulo de gestión } \\
\text { de servicio tecnológica }\end{array}$ \\
\hline Falta de procesos definidos & $\begin{array}{l}\text { Definición del mapa de procesos. } \\
\text { Levantamiento interno de procesos }\end{array}$ \\
\hline $\begin{array}{l}\text { Falta de aseguramiento de } \\
\text { servicio continuo }\end{array}$ & Base de Datos centralizada \\
\hline $\begin{array}{l}\text { Falta de aseguramiento de la } \\
\text { calidad y seguridad de sistema }\end{array}$ & $\begin{array}{l}\text { Gestión de la calidad } \\
\text { Gestión de mejora continua }\end{array}$ \\
\hline
\end{tabular}

Como se puede observar en el resumen de falencias y posibles soluciones, la mayoría de soluciones están basadas en el modelo planteado por ITIL, debido a que el objetivo de la aplicación del modelo ITIL a la SUPERCOM es el de ofrecer servicios tecnológicos de forma eficiente eficaz, es decir, que exista el mejor servicio posible en forma ininterrumpida para todos los usuarios en el tiempo pactado. El modelo involucra al factor humano, a los procesos, la infraestructura tecnológica y los proveedores que apoyan al desarrollo de los procesos y operaciones de la organización. A propuesta de modelos de procesos inicia con nuevo mapa de procesos ver figura1. En el cual se integran los procesos que agregadores de valor y que son críticos para la operación de la institución. 


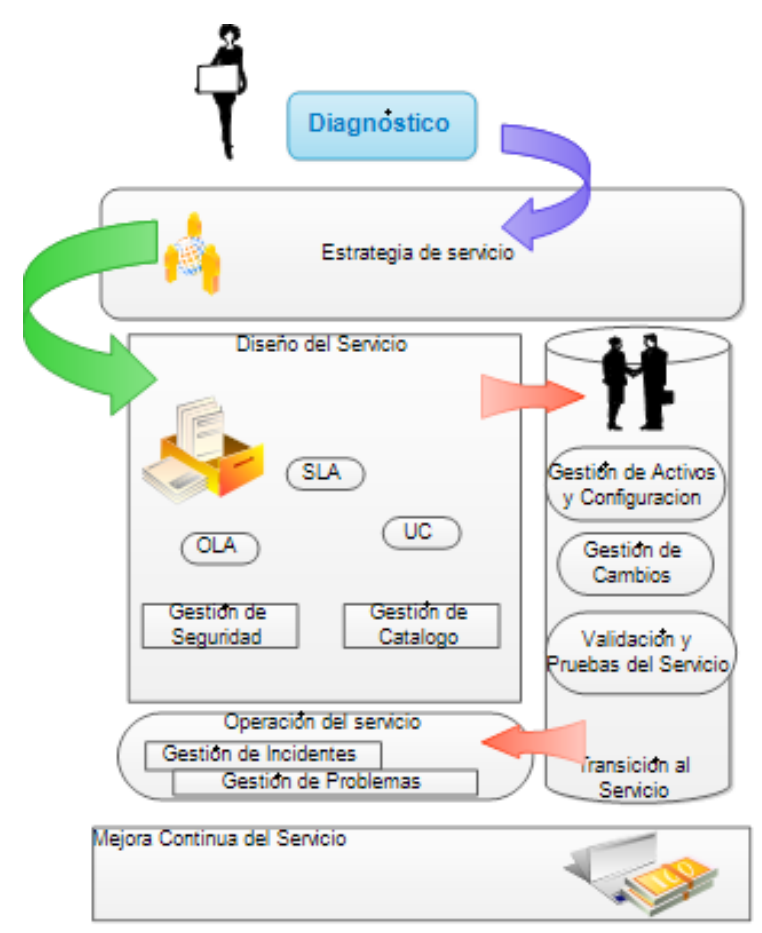

Figura.1. Mapa de procesos

\section{Diseño Modelo de Soporte Técnico}

En el Figura 2 se muestra un bosquejo del diseño que se realizara para la gestión de $\mathrm{TI}$ de la SUPERCOM, el cual cuenta con una mesa de ayuda donde son los encargados de gestionar las incidencias, problemas, versiones, configuraciones interactuando con el cliente y los técnicos para satisfacción de usuario final.

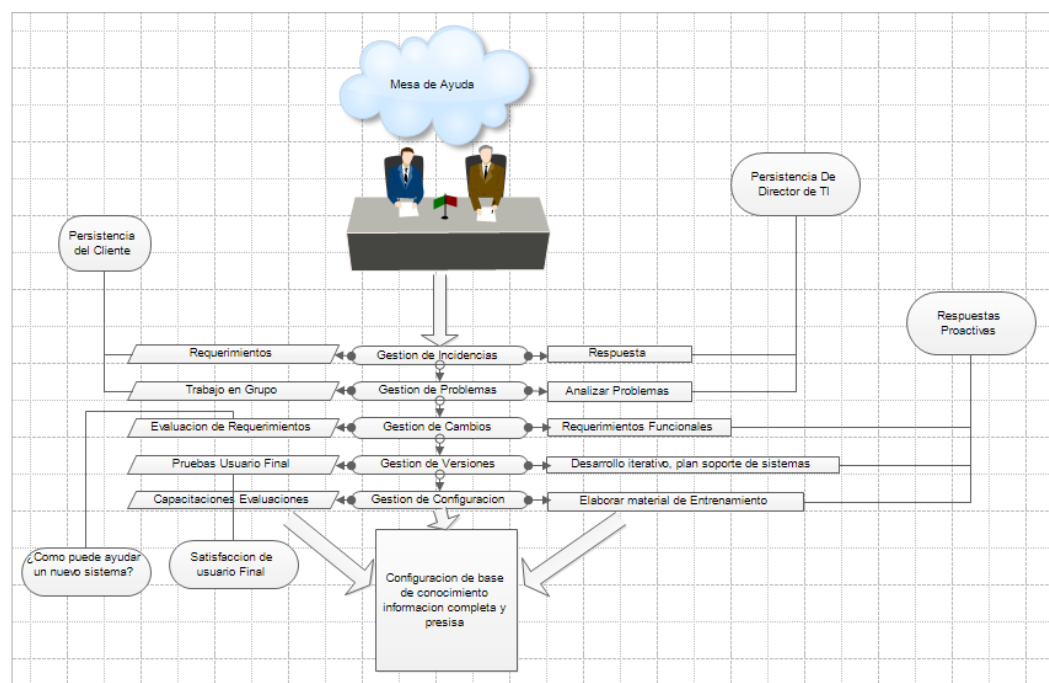

Figura.2. Bosquejo de diseño 
En la Figura 3 se muestra un diseño de la propuesta realizada para requerimientos de $\mathrm{TI}$ de la SUPERCOM, los cuales inicia con un aviso del usuario final sobre algún problema tecnológico lo cual lo puede realizar por, la WEB, telefónicamente, personalmente o vía correo electrónico, donde será atendido inmediatamente por la mesa de ayuda y asignara un técnico en caso que el técnico no pueda resolver el inconveniente será escalado a un especialista donde analizaran la gestión de problemas, o la gestión de incidentes dependiendo del problema que sea.

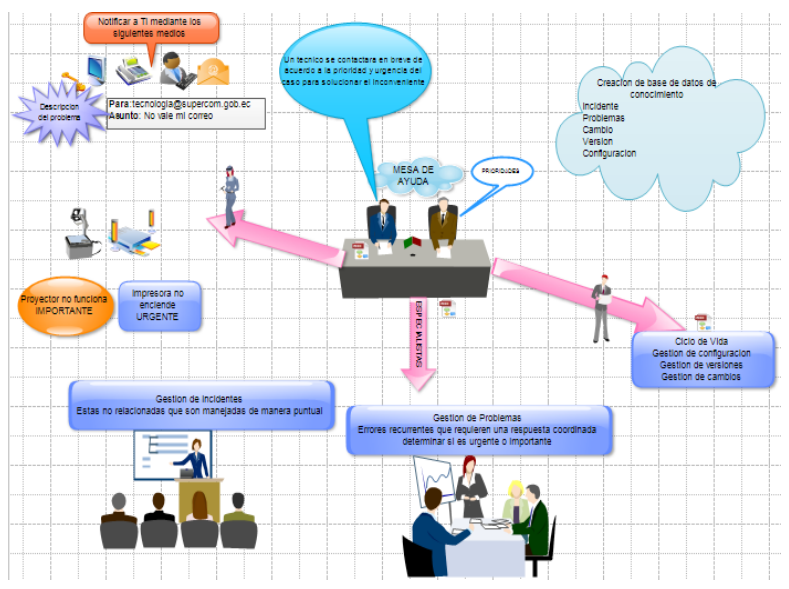

Figura.3. Diseño de requerimientos TI

En la Figura 4 se muestra un diseño de la propuesta del módulo de Soporte Técnico virtual El cual tendrá una base de datos centralizada con todas las intendencias Zonales que conforma la SUPERCOM teniendo las siguientes ventajas:

- Conocimiento Centralizado

- Evitar duplicidad de incidentes

- Reducción de costos operativos

- Sistema integrado de gestión de cambios

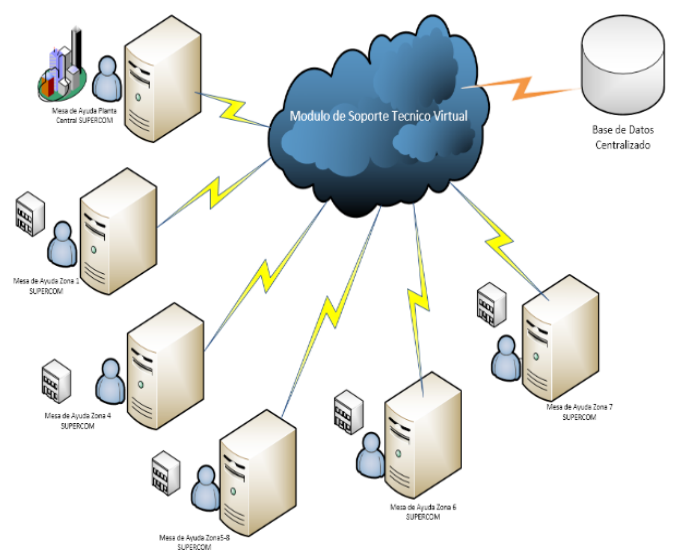

Figura.4. Diseño de la propuesta del módulo de soporte técnico. 


\section{IMPLEMETACION}

Para esto de dará unos pequeños detalles sobre la implementación del modelo de soporte técnico.

Se utiliza la metodología del modelo espiral ya que ofrece un aprovechando en el desarrollo del proyecto investigativo por ser incrementales e iterativos.

Para lo cual se va a requerir lo siguiente:

- Un servidor virtual en un ambiente ESXI 5.

- Sistema operativo Debían GNU/Linux 5 (64bit).

- Python 2.7.

- ODOO 9.

\section{Transmisión de voz sobre datos}

Joskowicz (2013) menciona que para lograr transmitir la voz sobre las redes de datos es necesario armar paquetes, un canal de voz tiene un flujo de bits dependiendo del codec utilizado. Sin embargo, se puede armar un paquete por cada flujo de voz, pero no es lo aconsejable debido a que esto generaría una sobrecarga (overhead) cabe mencionar algo muy importante que cada paquete requiere cabezas, en cambio para formar paquetes con una mínima sobrecarga pueden introducirse retrasos no muy aceptables.

\section{Datos}

Los datos se representan mediante manera simbólica y este es un valor que puede recibir un computador por diferentes medios de transmisión, los datos solos no pueden proporcionar información cuando estos forman una red de datos es ahí donde se considera una fuente de información.

\section{Redes de datos}

Las redes de datos tienen como objetivo:

- Compartir recursos, equipos, información y programas que se encuentran localmente y ubicados en distintos lugares.

- Brindar confiabilidad a la información disponiendo de alternativas de almacenamiento.

- Obtener una relación aceptable del costo y beneficio. 
- Transmitir información entre usuarios ubicados en distintos lugares geográficos de la manera más rápida y eficiente posible.

\section{Estrategia de gestión de servicios de voz y datos sobre internet}

Una estrategia de gestión de servicios de voz y datos sobre internet se puede definir como la forma de realizar el cambio de un proceso de los servicios orientados a la voz y datos dentro de una organización con la ayuda de sus metas u objetivos trazados, analizando la mejora de la capacidad del ancho de banda y controlando la calidad de servicio dentro de los parámetros que sean manejables para la organización sin afectar los tiempos de respuesta que se tengan dentro de las áreas involucradas, además de tomar en cuenta los dispositivos que la empresa dispone en el momento de realizar la investigación para en lo posterior sugerir mantener o actualizar.

\section{Operación de servicio}

Representa un proceso de ITIL para asegurar que los servicios se ofrezcan efectiva y eficientemente, esto incluye cumplir con los requerimientos de los usuarios, resolver los fallos de los servicios, arreglar problemas y llevar a cabo operaciones rutinarias, La rutina operación de servicio abarca los procesos siguientes: gestión de eventos, gestión de incidentes, cumplimiento de la solicitud, gestión de acceso, gestión de problemas, gestión de operaciones y gestión de instalaciones.

\section{Situación actual de la gestión de servicios de voz y datos sobre internet}

Actualmente los servicios de voz y datos que proporciona la corporación nacional de telecomunicaciones se encuentran vulnerados a través de sus terminales debido al encontrarse con malware que son activados por los hackers y esto provoca que la transmisión que lleva a los DNS (DomainNameSystem) se encuentre saturada provocando lentitud en el servicio de datos e internet en la ciudad de Quito, sin embargo, se activa manualmente la política de levantamiento de la transmisión que se encuentra dirigida hacia los servidores que se encuentran en Guayaquil.

Es decir, las transmisiones que se encuentran dentro del distrito metropolitano de Quito disponen de un enlace de respaldo, lo cual no sucede con los barrios que se encuentran a los alrededores de la ciudad y llevan el tráfico de voz y datos hacia las diferentes ciudades con el fin de que se encuentren conectadas y no se presenten percances cuando sufran algún corte de fibra y no se pueda respaldar el tráfico. Por tanto se quiere proponer una estrategia de gestión de servicios para las transmisiones que se encuentren saturadas y se pueda revisar e informar al área encargada para que esta proceda con el balanceo del tráfico respectivo en casa de que alguna de estas transmisiones se encuentre ocupando sobre el $85 \%$ de consumo y en caso de existir algún corte de fibra enviar a las cuadrillas a solventar el problema mientras el tráfico e encuentre balanceado, de tal manera, se necesita realizar el estudio de las transmisiones que se encuentran sin respaldo y proponer estrategias de ayuda y soporte. 


\section{RESULTADOS}

La SUPERCOM, ha mejorado los procesos de tecnologías de la información, ofreciendo servicios más eficientes a los usuarios, permitiendo establecer tiempos de respuesta para la resolución de incidentes enfocado en la gestión de las mejoras de los procesos de servicios basadas en ITIL.

Esto se lo demuestra en los índices de medición que proporciona el módulo de gestión de servicios y los objetivos planteados en esta investigación.

El modelo de servicios de proceso de tecnología de información, se basa en una estructura de acuerdo a lo propuesto por el marco de referencia de ITIL, donde se identifican las responsabilidades y el nivel de madurez de los procesos que tiene la SUPERCOM respecto al modelo propuesto por ITIL, además de las directrices para su diseño de desarrollo e implementación mediante la estrategia del servicio.

Por otro lado, se describen los flujos de información de los procesos en tecnologías de información de la SUPERCOM utilizando actualmente, para ello se realizó un inventario y como resultados se encontraron dos procesos principales para la Gestión de Catalogo de Servicio y Gestión de Incidencia.

Además, el modelo de mejora de los procesos de servicios tecnológicos de información se apoya en las buenas prácticas que propone ITIL, luego de lo cual se crea un departamento de tecnología de la información encargado de garantizar las excelencias operativas en la gestión de servicio de $\mathrm{TI}$.

\section{CONCLUSIONES}

Esta investigación propone una metodología para la implementación de un modelo de Soporte Técnico para la Gestión de Servicios Tecnológicos para mejorar la calidad en la gestión de los servicios tecnológicos, que se traduce en la eficiencia de la ejecución se los procesos y mayor control sobre la infraestructura y los servicios.

La investigación propuesta ofrece un conjunto de tareas cuyo cumplimiento permite llevar acabo procesos que basados en las normas y adaptabilidad que propone la metodología ITIL contribuyen al cumplimiento de los objetivos de los funcionarios de la SUPERCOM.

En este proyecto de investigación se plantearon objetivos específicos que han sido cumplidos a lo largo de este desarrollo e implementación del mismo. 


\section{REFERENCIAS BIBLIOGRÁFICAS}

Bergholz, S. P. (2011). Definición de gestión por procesos.

Definición de Proceso. (2011). Obtenido de http://definicion.de/proceso/.

Fernández, A., \& Llorens, F. (2011). Gobierno de las TI para universidades. Alicante: Conferencia de Rectores de las Universidades Españolas (CRUE).

ISO. (2017). ISO 9001:2017.

ISO/IEC. (2011). ISO/IEC 20000 Gestión de Servicios de TI. ISO/IEC.

SUPERCOM. (06 de 2017). Plan Estrategico Institucional 2014. Quito: SUPERCOM. Obtenido de http://www. supercom.gob.ec/images/d/lotaip/julio/pei.pdf 